\title{
Effect of inhomogeneous surface disorder on the superheating field of superconducting RF cavities
}

\author{
Vudtiwat Ngampruetikorn $\oplus^{*}$ and J. A. Sauls ${ }^{\dagger}$ \\ Center for Applied Physics \& Superconducting Technologies, Department of Physics \& Astronomy, \\ Northwestern University, Evanston, Illinois 60208, USA \\ and Fermi National Accelerator Laboratory, Batavia, Illinois 60510-5011, USA
}

(Received 10 September 2018; revised manuscript received 28 February 2019; published 29 August 2019)

\begin{abstract}
Recent advances in the surface treatments of niobium superconducting radio-frequency (SRF) cavities have led to substantially increased $Q$ factors and a maximum surface field. This poses theoretical challenges to identify the mechanisms responsible for such performance enhancements. We report theoretical results for the effects of inhomogeneous surface disorder on the superheating field-the surface magnetic field above which the Meissner state is globally unstable. We find that inhomogeneous disorder, such as that introduced by the infusion of nitrogen into the surface layers of niobium SRF cavities, can increase the superheating field above the maximum for superconductors in the clean limit or with homogeneously distributed disorder. Homogeneous disorder increases the penetration of the screening current, but also suppresses the maximum supercurrent. Inhomogeneous disorder in the form of an impurity diffusion layer biases this trade-off by increasing the penetration of the screening currents into cleaner regions with larger critical currents, thus limiting the suppression of the screening current to a thin dirty region close to the surface. Our results suggest that the impurity diffusion layers play a role in enhancing the maximum accelerating gradient of nitrogen-treated niobium SRF cavities.
\end{abstract}

DOI: 10.1103/PhysRevResearch.1.012015

Introduction. Niobium superconducting radio-frequency (SRF) cavities are the most energy-efficient engineered oscillators [1], with quality factors exceeding $2 \times 10^{11}$. SRF cavities operate at microwave frequencies and are the technology of choice for converting electromagnetic energy into highenergy particle beams, as well as intense photon sources, with applications ranging from fundamental particle physics experiments, atomic and molecular structure studies of condensed matter, to structural studies of biological materials for applications in biology and medicine [2]. The exceptional coherence properties of SRF cavities make them strong candidates for quantum memories [3-5]. The physical limits to the performance of these cavity oscillators are therefore of both fundamental and practical importance. To address the limits of the performance of SRF cavity oscillators, we must understand how superconductors fail in the presence of intense electromagnetic (EM) fields. In this Rapid Communication we address the effects of impurities infused into the current-carrying superconducting layer of an SRF cavity. Our results for the maximum sustainable surface field are relevant to recent, and possible future, experimental advances

\footnotetext{
*Present address: Initiative for the Theoretical Sciences, The Graduate Center, CUNY, New York, NY 10016, USA; vngampruetikorn@gc.cuny.edu

†sauls@northwestern.edu

Published by the American Physical Society under the terms of the Creative Commons Attribution 4.0 International license. Further distribution of this work must maintain attribution to the author(s) and the published article's title, journal citation, and DOI.
}

in the maximum field gradient of SRF cavities [6,7], which determines the transfer of electromagnetic field energy to charged particle beams.

Type-II superconductors admit two thermodynamic phases in the presence of an external magnetic field $H$. The Meissner state is the equilibrium state for fields below a lower critical field $H<H_{c_{1}}$, while the Abrikosov state, characterized by the penetration of quantized flux into the bulk of the superconductor, is thermodynamically stable for fields $H_{c_{1}}<H<H_{c_{2}}$, where $H_{c_{2}}$ is the critical field above which the superconductor becomes normal for any temperature $T \leqslant T_{c}$. Superconductors in the Meissner state exhibit perfect diamagnetism by generating an internal field, that exactly screens the external field. The source of the screening field is a dissipationless supercurrent, "screening current," confined to the vacuumsuperconductor interface. The screening current penetrates into the superconductor over a mesoscopic length scale, the London penetration depth $\lambda_{L}$, which is sensitive to disorder. The magnitude of the screening current increases linearly with the applied field until the cost in kinetic energy of maintaining perfect diamagnetism is outweighed by the reduction in Gibbs energy via flux penetration into the bulk. For type-II superconductors flux is quantized in units of $\Phi_{0}=h c / 2 e$ and confined in tubes of radius of order $\lambda_{L}$, and the lower critical field for flux penetration is $H_{c_{1}}=\Phi_{0} / 2 \pi \lambda_{L}^{2}$, which for SRF-grade $\mathrm{Nb}$ is typically of order $H_{c_{1}} \approx 170 \mathrm{mT}$, or an accelerating field of $E_{\mathrm{ac}} \approx 25 \mathrm{MV} / \mathrm{m}$.

Above $H_{c_{1}}$ the Abrikosov state, with an array of quantized flux lines, is the thermodynamically stable phase. The motion of quantized flux generates Joule losses and is detrimental to the performance of SRF cavities for particle acceleration. Understanding, and thus engineering, materials properties and 
physical processes governing the breakdown of the Meissner state is crucial for developing strategies to improve the performance of SRF cavities.

One key feature is that the Meissner state can be maintained for fields higher than $H_{c_{1}}$ as a metastable phase, made possible by a surface energy barrier to flux penetration [8]. At sufficiently high field, the so-called superheating field $H_{\text {sh }}>H_{c_{1}}$, the surface barrier vanishes, and flux lines proliferate, leading to dissipation under RF excitation.

The superheating field depends on the geometry of the vacuum-superconductor interface as well as the spatial distribution of disorder within the region of the screening currents. For a planar half-space geometry the effects of homogeneous disorder and engineered multilayer superconductor-insulator structures have been studied [9-15]. The main results are that homogeneous disorder increases the penetration depth, but reduces the critical current, leading to a modest enhancement of the superheating field at low temperatures [9]. For a bilayer material consisting of a dirty layer on top of a clean superconductor, it was shown in Ref. [15] that the superheating field can exceed that of either material. The results are based on a phenomenological theory whose range of validity is far from the operating conditions of SRF cavities. The superheating field may also be increased by introducing insulating layers to retard flux line penetration $[10,11,13,15]$. Here, we report the results of a theoretical investigation of the effects of an impurity diffusion layer, i.e., a smoothly varying, coarse-grained impurity density within the region of the screening currents, on SRF cavities such as nitrogen infused into niobium [7].

In general, it is technically challenging to obtain quantitative predictions for the superheating field as one must consider the stability of the Meissner state to inhomogeneous fluctuations of the order parameter and charge currents, as well as nucleation of vortices around impurities, inclusions, or sharp structures at the vacuum-superconductor interface. Here, we consider the upper limit for the superheating field, which is the lowest surface field at which the supercurrent density reaches the local critical current density at some point within the screening region. This condition provides an upper bound to the superheating field since any increase in the local condensate momentum-equivalently the local vector potential—cannot increase the supercurrent density. At the superheating field the Meissner state is unstable to arbitrarily small perturbations of the order parameter and EM field. For extreme type-II superconductors, this approach is equivalent to the stability condition with respect to inhomogeneous fluctuations of the order parameter and the associated EM response [16].

Type-II superconductors are characterized by the Ginzburg-Landau parameter $\kappa=\lambda_{L} / \xi \geqslant 1 / \sqrt{2}$, where $\lambda_{L}$ denotes the London penetration depth and $\xi$ is the superconducting coherence length. Pure $\mathrm{Nb}$ is weakly type II with $\kappa \approx 1$. However, disorder leads to increased field penetration with $\kappa \gg 1$ in the "dirty limit," $\hbar / \tau \gg \Delta$, where $\tau$ is the mean quasiparticle-impurity collision time. In this strong type-II limit quasiparticles and Cooper pairs respond locally to a nearly uniform EM field.

Here, we consider superconductors in the strong type-II limit occupying the half space $x>0$ in the presence of an external magnetic field, $\mathbf{H}_{\mathrm{a}}=H_{a} \hat{\mathbf{z}}$, applied parallel to the

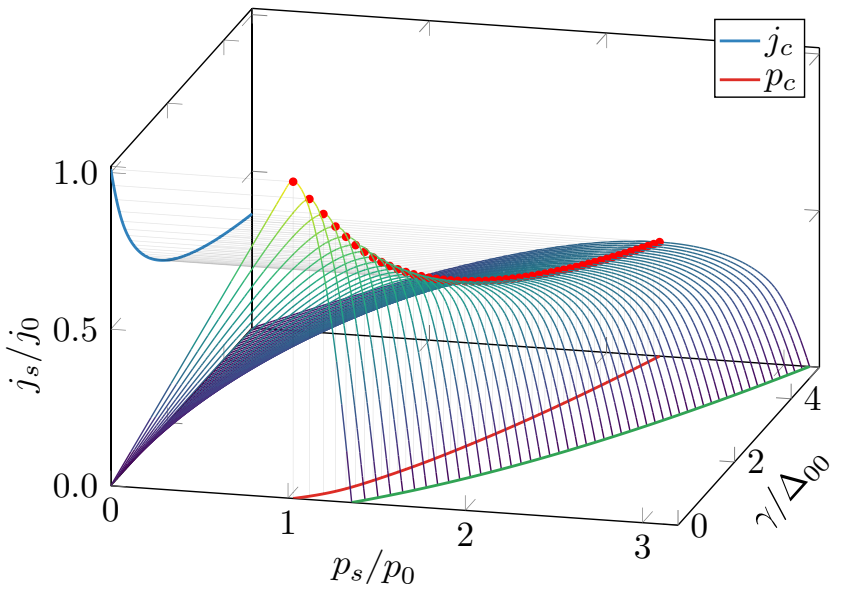

FIG. 1. The supercurrent density $j_{s}$ as a function of condensate momentum $p_{s}$ and impurity scattering rate $\gamma$ at $T=0$. For fixed $\gamma$, the critical current $j_{c}$ and condensate momentum $p_{c}$ correspond to the values at which $j_{s}$ is maximum (red solid circles). The critical current $j_{c}$ decreases with increasing $\gamma$ (blue line), whereas the critical condensate momentum $p_{c}$ increases with increasing $\gamma$ (red line). For $p_{s}>p_{c}$, the Meissner current is unstable.

vacuum-superconductor interface. We include the effects of an impurity diffusion layer on the current response into the quasiparticle-impurity scattering rate and pairing self-energy. Based on Eilenberger's quasiclassical transport theory [17], we compute the superfluid momentum $\mathbf{p}_{s}=p_{s}(x) \hat{\mathbf{y}}$, screening supercurrent $\mathbf{j}_{s}=j_{s}(x) \hat{\mathbf{y}}$, and local magnetic induction $\mathbf{B}=$ $B(x) \hat{\mathbf{z}}$ self-consistently. The superheating field $H_{\mathrm{sh}}$ is the value of the surface field $B(0)$ at which the supercurrent density reaches the local critical value anywhere in the screening region of the superconductor, i.e., $\min _{x}\left[j_{c}(x)-\left|j_{s}(x)\right|\right]=0$. Note that the critical current density $j_{c}(x)$ is a function of position due to the inhomogeneous impurity diffusion layer (Fig. 1).

Methods. For a superconductor in the strong type-II limit, with an impurity diffusion layer that also varies on a length scale much longer than $\xi$, we develop the Eilenberger transport equation as a perturbation expansion in the small ratios, $\epsilon \in\left\{\xi / \lambda_{L}, \xi / \zeta\right\}$, where $\zeta$ is the characteristic penetration length of the impurity diffusion layer [18]. To leading order in $\epsilon$ the current response is determined by the retarded quasiclassical propagator obtained from the homogeneous solution of the quasiclassical transport equation, but evaluated with the Doppler-shifted excitation spectrum determined by the local condensate momentum $p_{s}(x)$ and the local impurity self-energies [18],

$$
\begin{aligned}
\widehat{\mathfrak{G}}(\hat{\mathbf{p}}, \varepsilon, x) & =-\pi \frac{\left[\tilde{\varepsilon}(\varepsilon, x)-\mathbf{v}_{f} \cdot \mathbf{p}_{s}(x)\right] \widehat{\tau}_{3}-\tilde{\Delta}(\varepsilon, x)\left(i \sigma_{y} \widehat{\tau}_{1}\right)}{\sqrt{|\tilde{\Delta}(\varepsilon, x)|^{2}-\left[\tilde{\varepsilon}(\varepsilon, x)-\mathbf{v}_{f} \cdot \mathbf{p}_{s}(x)\right]^{2}}} \\
& \equiv-\pi\left[\mathfrak{G}(\hat{\mathbf{p}}, \varepsilon, x) \widehat{\tau}_{3}-\mathfrak{F}(\hat{\mathbf{p}}, \varepsilon, x)\left(i \sigma_{y} \widehat{\tau}_{1}\right)\right],
\end{aligned}
$$

where $\widehat{\tau}_{i}$ and $\sigma_{i}$ denote the Pauli matrices in particle-hole and spin space, respectively, $\hat{\mathbf{p}}$ is the direction defined by a point on the Fermi surface, $\mathbf{p}=p_{f} \hat{\mathbf{p}}$, and $\mathbf{v}_{f}=v_{f} \hat{\mathbf{p}}$ is the corresponding Fermi velocity. In the absence of vortices the superfluid momentum can be related to the vector potential 
via $\mathbf{p}_{s}=(-e / c) \mathbf{A}$, where we have fixed the gauge by absorbing the gradient of the phase of the condensate into $\mathbf{A}$. The diagonal and off-diagonal propagators $\mathfrak{G}$ and $\mathfrak{F}$ encode the information about the local equilibrium quasiparticle and Cooper pair spectral functions.

The impurity renormalized quasiparticle excitation energy and off-diagonal pairing energy can then be expressed as

$$
\begin{aligned}
\tilde{\varepsilon}(\varepsilon, x) & =\varepsilon+\gamma(x)\langle\mathfrak{G}(\hat{\mathbf{p}}, \varepsilon, x)\rangle_{\hat{\mathbf{p}}}, \\
\tilde{\Delta}(\varepsilon, x) & =\Delta(x)+\gamma(x)\langle\mathfrak{F}(\hat{\mathbf{p}}, \varepsilon, x)\rangle_{\hat{\mathbf{p}}},
\end{aligned}
$$

where $\langle\cdots\rangle_{\hat{\mathbf{p}}}$ denotes an angular average over the Fermi surface and $\gamma(x)$ is the local impurity scattering rate. The order parameter $\Delta(x)$ satisfies the mean-field BCS gap equation $\Delta(x)=\frac{g}{2}-\int d \varepsilon \tanh \frac{\varepsilon}{2 T} \operatorname{Im}\langle\mathfrak{F}(\hat{\mathbf{p}}, \varepsilon, x)\rangle_{\hat{\mathbf{p}}}$, where $g$ is the pairing interaction, and the integration extends over the lowenergy bandwidth set by the Debye energy.

The solution for the field penetration into the inhomogeneous Meissner region of the superconductor is obtained from the local current response, which is in general a nonlinear function of the condensate momentum $\mathbf{p}_{s}(x)$ combined with Ampère's equation. The latter equation can be expressed as

$$
\partial_{x}^{2} \mathbf{p}_{s}(x)-\frac{4 \pi e}{c^{2}} \mathbf{j}_{s}\left[\mathbf{p}_{s}(x), \gamma(x)\right]=\mathbf{0},
$$

where the supercurrent is obtained from the local solution for the quasiclassical propagator (see Fig. 1),

$$
\mathbf{j}_{s}(x)=-e N_{f} \int d \varepsilon \tanh \frac{\varepsilon}{2 T}\left\langle\mathbf{v}_{f} \mathcal{A}(\hat{\mathbf{p}}, \varepsilon, x)\right\rangle_{\hat{\mathbf{p}}},
$$

where $N_{f}=p_{f}^{2} / 2 \pi^{2} \hbar^{3} v_{f}$ is the normal-state density of states, per spin, at the Fermi level. The Meissner current sums the charge current contributions from the states comprising both the negative energy condensate, as well as thermally excited Bogoliubov quasiparticles, governed by the angle-resolved spectral function $\mathcal{A}(\hat{\mathbf{p}}, \varepsilon ; x) \equiv \frac{-1}{\pi} \operatorname{Im} \mathfrak{G}(\hat{\mathbf{p}}, \varepsilon ; x)$ and the thermal distribution function $\Phi(\varepsilon)=\tanh (\varepsilon / 2 T)$.

To determine the magnetic field distribution in the superconductor, we find the self-consistent condensate momentum distribution $p_{s}(x)$ that determines the supercurrent $j_{s}(x)$ [Eq. (4)] and is also the solution of Ampère's law [Eq. (3)]. Ampère's law is also supplemented by boundary conditions at the surface and the asymptotic condition far from the vacuumsuperconductor interface,

$$
\nabla \times\left.\mathbf{p}_{s}(x)\right|_{x=0}=(-e / c) \mathbf{H}_{\mathrm{a}}, \quad \text { and } \quad \lim _{x \rightarrow \infty} \mathbf{p}_{s}(x)=\mathbf{0} .
$$

The asymptotic condition reflects the fact that the Meissner state exhibits perfect diamagnetism. Equations (1)-(5) constitute a closed set of equations which are solved selfconsistently. The local magnetic induction can then be computed directly from $\mathbf{B}(x)=(-c / e) \partial_{x} p_{s}(x) \hat{\mathbf{z}}$.

In order to determine the superheating field we first solve Eqs. (1)-(5) self-consistently for fixed temperature $T$, external field $H_{a}$, and impurity distribution $\gamma(x)$, which yields the self-consistently determined spatial profiles for the condensate momentum $p_{s}(x)$ and Meissner screening current $j_{s}(x)$. The spatial profile of the magnetic field is obtained from the condensate momentum $B(x)=(-c / e) \partial_{x} p_{s}(x)$. The superheating field is the surface field, $B(0)=H_{\text {sh }}$, at which the

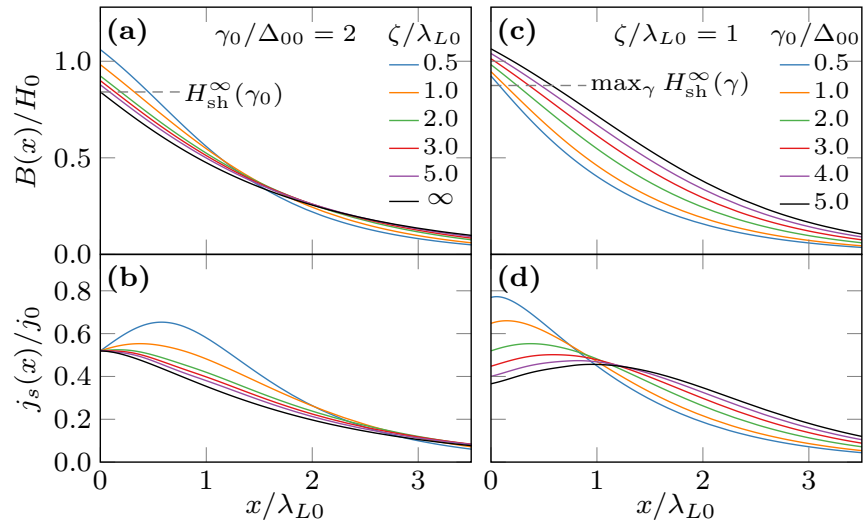

FIG. 2. The magnetic field and current density profiles $B(x)$ and $j_{s}(x)$ at the superheating field for temperature $T=0$, various impurity diffusion lengths $\zeta$, and surface scattering rate $\gamma_{0}$ shown in the legend. (a) $B(x)$ in units of the zero-temperature, clean-limit critical field, $H_{0}=\sqrt{4 \pi N_{f} \Delta_{00}^{2}}$ at $\gamma_{0} / \Delta_{00}=2$, where $\Delta_{00}=1.78 T_{c}$ is the zero-temperature BCS gap in the clean limit. The superheating field $H_{\mathrm{sh}}=B(0)$ increases with decreasing diffusion length $\zeta$, and exceeds the superheating field for the case of homogeneous disorder with scattering rate $\gamma_{0}$ (dashed line). (b) $j_{s}(x)$ in units of the zerotemperature, clean-limit critical current $j_{0}=e n \Delta_{00} / p_{f}$ for $\zeta$ and $\gamma_{0}$ shown in (a). The current density builds up away from the surface as $\zeta$ decreases, leading to larger total screening currents (the area under the curves) and thus higher superheating fields. (c) and (d) Same as (a) and (b), but for a fixed impurity diffusion length of $\zeta / \lambda_{L 0}=1$ as a function of surface scattering rate $\gamma_{0}$, shown in the legend. The superheating field $H_{\mathrm{sh}}=B(0)$ exceeds the theoretical maximum for the case of homogeneous disorder [9] (dashed line), over the whole range of $\gamma_{0}$.

supercurrent and the superfluid momentum reach local critical values anywhere in the screening region.

For concreteness we model the impurity diffusion layer as exponential decay from the vacuum-superconducting interface, $n_{\mathrm{imp}}(x)=n_{0} \exp (-x / \zeta)$, or equivalently a local scattering rate of the form

$$
\gamma(x)=\gamma_{0} e^{-x / \zeta}
$$

where $\gamma_{0}$ denotes the impurity scattering rate at $x=0$ and $\zeta$ is the impurity diffusion length. Similar results are obtained based on a Gaussian diffusion layer. This model qualitatively captures the impurity distribution in nitrogen-treated SRF cavities, i.e., high-impurity concentration near the surface and very low-impurity concentration in the bulk [7]. We confine our analysis to diffusion lengths that are large compared to the coherence length, $\zeta \gg \xi$, so that we can evaluate the propagator with the locally homogeneous solution in Eq. (1). In this model the condensate momentum first reaches the critical value at the surface, i.e., the superheating condition is given by $p_{s}(0)=p_{c}(0)$, where $p_{c}(0)$ is the critical condensate momentum determined by the maximum scattering rate $\gamma_{0}$.

Results. Figures 2(a) and 2(b) show the magnetic field and current density profiles at the superheating field for a scattering rate at the surface, $\gamma_{0} / \Delta_{00}=2$, where $\Delta_{00}$ is the excitation gap at $T=0$ in the clean limit. We present results for impurity diffusion lengths ranging from the homogeneous limit, $\zeta \rightarrow \infty$, to $\zeta / \lambda_{L 0}=0.5$, scaled in units of the clean- 

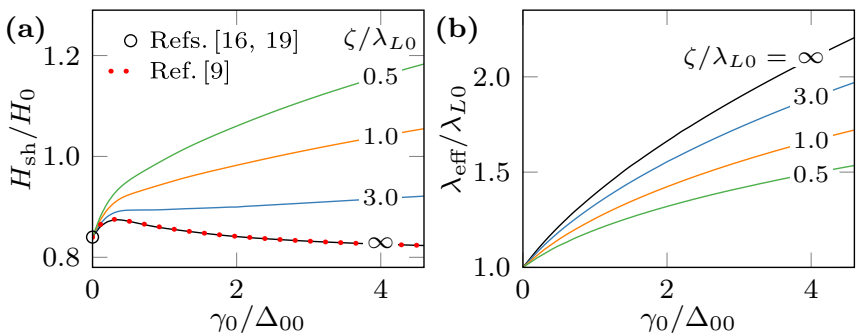

FIG. 3. The superheating field $H_{\mathrm{sh}}$ and effective penetration depth $\lambda_{\text {eff }}$ in superconductors with an impurity diffusion layer [Eq. (6)] as functions of the surface scattering rate $\gamma_{0}$ for various impurity diffusion lengths $\zeta$ shown in the legend. We compare our results for the superheating field with previous calculations in the clean limit [16,19] (black open circle) and for homogeneous disorder [9] (red circles).

limit, $T=0$, zero-field London penetration depth, $\lambda_{L 0}=$ $1 /\left(8 \pi e^{2} v_{f}^{2} N_{f} / 3 c^{2}\right)^{\frac{1}{2}}$, but restricted to $\zeta \gg \xi_{0}$. Figure 2(a) shows that the superheating field, given by the field at $x=0$, increases with decreasing impurity diffusion length, and exceeds the superheating field for the case of homogeneous disorder with scattering rate $\gamma / \Delta_{00}=2$ (dashed line).

To understand how an inhomogeneous impurity distribution leads to an increase in the superheating field, consider the current density profiles shown in Fig. 2(b). At the superheating field the current density at $x=0$ is equal to the local critical current density, which is determined by $\gamma_{0}$ in each case. However, away from the surface a shorter impurity diffusion length results in a reduced impurity density and therefore larger current density for a given value of the local condensate momentum. Indeed, for sufficiently short impurity diffusion lengths the current density peaks at a finite distance from the vacuum-superconductor interface, resulting in a larger integrated screening current, $J=\int_{0}^{\infty} d x j(x)$, and thus a higher superheating field.

Figures 2(c) and 2(d) show the magnetic field and current density profiles at the superheating field for a fixed impurity diffusion length $\zeta / \lambda_{L 0}=1$, for a range of maximum impurity scattering rates $\gamma_{0}$. In Fig. $2(\mathrm{c})$ the magnetic field penetrates deeper into the superconductor with increasing impurity scattering at the surface, and the superheating field increases above the absolute maximum superheating field for homogeneous disorder [9] (dashed line). The screening current penetrates deeper further into the superconductor, but is suppressed for $x \lesssim \lambda_{L 0}$ [Fig. 2(d)]. However, the local suppression of the current near the surface is overcompensated by the increase in the screening current for $x \gtrsim \lambda_{L 0}$ over a longer effective penetration depth, leading to an increase in the superheating field.

Figure 3 summarizes our results for the superheating field in impurity diffusion layers at $T=0$. Disorder affects the superheating field via two competing mechanisms. First, the effective penetration depth, defined as $\lambda_{\text {eff }} \equiv$ $B(0)^{-1} \int_{0}^{\infty} d x B(x)$, increases with disorder [Fig. 3(b)]. As a result, the screening current penetrates deeper into the superconductor, increasing the total screening current, and as a result the superheating field. Second, impurity scattering suppresses the critical current and superheating field (cf. Fig. 1).
For homogeneous disorder the increase in the effective penetration depth is dominant at low scattering rates, while the suppression of supercurrent dominates at higher scattering rates. As a result, the superheating field develops a peak at a relatively modest level of disorder, $\gamma_{0} / \Delta_{00} \approx 0.3$ [Fig. 3(a)] for $\zeta / \lambda_{L 0}=\infty$, with $H_{\text {sh }} \approx 0.87 H_{0}$. However, in impurity diffusion layers the suppression of supercurrent is confined to the region near the surface $x \lesssim \zeta$, while due to a longer effective penetration depth [cf. Fig. 2(d)], the screening current shifts to the relatively clean region with $x \gtrsim \zeta$. This results in a superheating field that increases with the surface scattering rate, as shown in Fig. 3(a) for diffusion lengths $\zeta / \lambda_{L 0} \lesssim 3.0$ [20].

Our analysis for the superheating field based on the local critical depairing current is equivalent to the stability analysis of the Meissner state presented in Refs. [9,16,19]. Indeed, our results agree with the previous calculations based on analyses of the thermodynamic potential. In particular, we obtain $H_{\mathrm{sh}} / H_{0} \approx 0.84$ for clean type-II superconductors as reported in Refs. [9,16,19] [black open circle in Fig. 3(a)]. Our results also agree with those of Ref. [9] for the limit of homogeneous disorder [red data points in Fig. 3(a)].

So far we have considered the extreme type-II limit with $\kappa^{-1}=\xi / \lambda_{L} \rightarrow 0$. Niobium, the material of choice for SRF applications, is marginally type II in the clean limit with $\kappa \approx 1$ [21-23]. Cavity-grade niobium has surface disorder, and is treated with nitrogen impurities to increase performance, both of which increase the Ginzburg-Landau parameter, thus suppressing the corrections to our theory which are of order $\kappa^{-2}$ [18]. Thus, we believe this work provides new insight into the role of inhomogeneous disorder on the superheating field in nitrogen-infused niobium SRF cavities. Moreover, our results have implications for the other superconducting materials considered for SRF applications, such as $\mathrm{Nb}_{3} \mathrm{Sn}$ and $\mathrm{MgB}_{2}$, both of which are strongly type II with $\kappa \gtrsim 20$ [24,25].

Summary and outlook. We report a theoretical investigation based on a microscopic theory of inhomogeneous superconductors of the effects of impurity diffusion layers on the superheating field of SRF cavities, the limiting magnetic field beyond which the Meissner state is unstable. A key result is that the introduction of impurity diffusion layers, for example, by nitrogen infusion into niobium, can increase the superheating field of SRF cavities above the maximum value predicted for the homogeneous disorder model [9]. The underlying mechanism is the increase in screening current resulting from an increased field penetration depth which overcompensates suppression of the Meissner current in the relatively thin dirty region near the surface. Our results strongly suggest that impurity diffusion layers play a role in enhancing the maximum accelerating gradient of treated SRF cavities. Although the increase in the superheating field appears to be generic, the magnitude of the increase depends on specific impurity profiles, suggesting that it might be possible to further increase the superheating field by engineering disorder profiles.

While inhomogeneous impurity layers can enhance the performance of SRF cavities in accelerator applications, their effects in the low-field limit on photon coherence time, an important figure of merit in quantum information applications, are less clear. Impurity doping and infusion can result 
in a higher-quality factor [1,7] which tends to increase the coherence time, however, such surface preparations could also lead to a higher abundance of two-level systems which limits the quality factor, and thus coherence time, in low-field limits [3]. The effects of impurity diffusion layers on the lowfield performance of SRF cavities is an important research direction for the future.
Acknowledgments. We thank Anna Grassellino, Alex Romanenko, Mattia Checchin, and Martina Martinello for discussions on N-doped niobium SRF cavities which motivated this research. The research of the authors is supported by National Science Foundation Grant No. PHY-1734332 and the Northwestern-Fermilab Center for Applied Physics and Superconducting Technologies.
[1] A. Romanenko, A. Grassellino, A. C. Crawford, D. A. Sergatskov, and O. Melnychuk, Ultra-high quality factors in superconducting niobium cavities in ambient magnetic fields up to $190 \mathrm{mG}$, Appl. Phys. Lett. 105, 234103 (2014).

[2] H. Padamsee, 50 years of success for SRF accelerators-a review, Supercond. Sci. Technol. 30, 053003 (2017).

[3] A. Romanenko and D. I. Schuster, Understanding Quality Factor Degradation in Superconducting Niobium Cavities at Low Microwave Field Amplitudes, Phys. Rev. Lett. 119, 264801 (2017).

[4] Y. Y. Gao, B. J. Lester, Y. Zhang, C. Wang, S. Rosenblum, L. Frunzio, L. Jiang, S. M. Girvin, and R. J. Schoelkopf, Programmable Interference Between Two Microwave Quantum Memories, Phys. Rev. X 8, 021073 (2018).

[5] A. Romanenko, R. Pilipenko, S. Zorzetti, D. Frolov, M. Awida, S. Posen, and A. Grassellino, Three-dimensional superconducting resonators at $T<20 \mathrm{mK}$ with the photon lifetime up to $\tau=2$ seconds, arXiv: 1810.03703 .

[6] A. Grassellino, A. Romanenko, D. Sergatskov, O. Melnychuk, Y. Trenikhina, A. Crawford, A. Rowe, M. Wong, T. Khabiboulline, and F. Barkov, Nitrogen and argon doping of niobium for superconducting radio frequency cavities: A pathway to highly efficient accelerating structures, Supercond. Sci. Technol. 26, 102001 (2013).

[7] A. Grassellino, A. Romanenko, Y. Trenikhina, M. Checchin, M. Martinello, O. S. Melnychuk, S. Chandrasekaran, D. A. Sergatskov, S. Posen, A. C. Crawford, S. Aderhold, and D. Bice, Unprecedented quality factors at accelerating gradients up to $45 \mathrm{MVm}^{-1}$ in niobium superconducting resonators via low temperature nitrogen infusion, Supercond. Sci. Technol. 30, 094004 (2017).

[8] C. P. Bean and J. D. Livingston, Surface Barrier in Type-II Superconductors, Phys. Rev. Lett. 12, 14 (1964).

[9] F. P.-J. Lin and A. Gurevich, Effect of impurities on the superheating field of type-II superconductors, Phys. Rev. B 85, 054513 (2012).

[10] T. Kubo, Y. Iwashita, and T. Saeki, Radio-frequency electromagnetic field and vortex penetration in multilayered superconductors, Appl. Phys. Lett. 104, 032603 (2014).

[11] S. Posen, M. K. Transtrum, G. Catelani, M. U. Liepe, and J. P. Sethna, Shielding Superconductors with Thin Films as Applied to rf Cavities for Particle Accelerators, Phys. Rev. Appl. 4, 044019 (2015).

[12] A. Gurevich, Maximum screening fields of superconducting multilayer structures, AIP Adv. 5, 017112 (2015).
[13] D. B. Liarte, M. K. Transtrum, and J. P. Sethna, GinzburgLandau theory of the superheating field anisotropy of layered superconductors, Phys. Rev. B 94, 144504 (2016).

[14] D. B. Liarte, S. Posen, M. K. Transtrum, G. Catelani, M. Liepe, and J. P. Sethna, Theoretical estimates of maximum fields in superconducting resonant radio frequency cavities: Stability theory, disorder, and laminates, Supercond. Sci. Technol. 30, 033002 (2017).

[15] T. Kubo, Multilayer coating for higher accelerating fields in superconducting radio-frequency cavities: A review of theoretical aspects, Supercond. Sci. Technol. 30, 023001 (2017).

[16] G. Catelani and J. P. Sethna, Temperature dependence of the superheating field for superconductors in the high- $\kappa$ London limit, Phys. Rev. B 78, 224509 (2008).

[17] G. Eilenberger, Transformation of Gorkov's equation for type II superconductors into transport-like equations, Z. Phys. 214, 195 (1968).

[18] See Supplemental Material at http://link.aps.org/supplemental/ 10.1103/PhysRevResearch.1.012015 for the derivation of Eq. (1) and the expansion of the quasiclassical transport equations in terms of $\epsilon=\left\{\xi / \zeta, \xi / \lambda_{L}\right\}$.

[19] V. P. Galaiko, Stability limits of the superconducting state in a magnetic field for superconductors of the second kind, $\mathrm{Zh}$. Eksp. Teor. Fiz. 50, 717 (1966) [Sov. Phys. JETP 23, 475 (1966)].

[20] For the surface scattering rates $\gamma_{0}$ shown in Fig. 3, the superheating field reaches a maximum at $\zeta^{*} \lesssim 0.4 \lambda_{L 0}$. However, the effects of inhomogeneous disorder varying on such a short length scale are just outside the scope of our current approximation. A more quantitative determination of the optimal $\zeta^{*}$ requires a full nonlocal theory for the superheating field.

[21] T. F. Stromberg and C. A. Swenson, Negative Surface FreeEnergy Effects in Superconducting Niobium, Phys. Rev. Lett. 9, 370 (1962).

[22] B. W. Maxfield and W. L. McLean, Superconducting Penetration Depth of Niobium, Phys. Rev. 139, A1515 (1965).

[23] D. K. Finnemore, T. F. Stromberg, and C. A. Swenson, Superconducting properties of high-purity niobium, Phys. Rev. 149, 231 (1966).

[24] T. P. Orlando, E. J. McNiff, S. Foner, and M. R. Beasley, Critical fields, Pauli paramagnetic limiting, and material parameters of $\mathrm{Nb}_{3} \mathrm{Sn}$ and $\mathrm{V}_{3} \mathrm{Si}$, Phys. Rev. B 19, 4545 (1979).

[25] B. B. Jin, N. Klein, W. N. Kang, H.-J. Kim, E.-M. Choi, S.-I. Lee, T. Dahm, and K. Maki, Energy gap, penetration depth, and surface resistance of $\mathrm{MgB}_{2}$ thin films determined by microwave resonator measurements, Phys. Rev. B 66, 104521 (2002). 